\title{
La participación de mujeres indígenas rurales para fortalecer la gobernanza comunitaria
}

\author{
Selmira Flores, Kristen Evans, Anne M. Larson, Alejandro Pikitle y Roberto Marchena
}

\section{Mensajes principales}

- Las mujeres miskitu y mayangna de las comunidades rurales que dependen del bosque de la Región Autónoma de la Costa Caribe Norte de Nicaragua continúan con poca presencia y en desventaja en los espacios de representación en las instancias de toma de decisiones, debido en parte a la débil gobernanza comunitaria. Fortaleciendo la participación de las mujeres se mejora la gobernanza comunitaria.

- Las normativas culturales continúan privilegiando a los varones. El camino hacia la equidad de género exige espacios para que las mujeres reflexionen sobre su rol en la gobernanza forestal comunitaria, pero a la vez requiere del compromiso de parte de los varones para respetar y respaldar sus propuestas.

- El monitoreo participativo sobre la gobernanza abrió un espacio para que las mujeres expresaran sus preocupaciones sobre los problemas y conflictos en el manejo de los recursos naturales y también abrió esperanzas de cambio.

\section{Introducción}

A pesar de las reformas legales y esfuerzos institucionales para mejorar la participación de las mujeres en los procesos de gobernanza, las mujeres de cinco comunidades indígenas que estudiamos en la Región Autónoma de la Costa Caribe Norte de Nicaragua (RACCN), no participan de manera significativa en la toma de decisiones sobre los asuntos comunitarios vinculados al manejo de los recursos naturales². La gobernanza deficiente y las barreras culturales continúan restringiendo la participación de las mujeres. Este planteamiento se deriva de los resultados de la investigación participativa implementada durante 2014 y 2015 por el instituto de investigación Nitlapan de la Universidad Centroamericana y el Centro para la Investigación Forestal Internacional (CIFOR).

Durante el transcurso de dos años observamos la dinámica de género por medio de métodos participativos: monitoreo participativo, reflexión colectiva, entrevistas y observación participante. Dos resultados de este proceso indican que, primero, las mujeres indígenas de la comunidad rural siguen siendo minorías sin voz en las estructuras de los gobiernos comunales y territoriales donde se toman decisiones (Pikitle,

Por ejemplo, la Ley 648 de Igualdad de Derechos y Oportunidades de 2008, la Política de Género de la RAAN (ahora RACCN) de 2010 (Mairena et al. 2012), y la Ley Electoral (No. 331, con sus reformas No.790/2012) que requiere que $50 \%$ de los candidatos sean mujeres.

2 La participación de las mujeres indígenas en las comunidades rurales suele circunscribirse a los roles designados culturalmente de acuerdo al papel que se concibe para ellas. Las mujeres se responsabilizan en la comunidad por la organización de actividades para la iglesia, la salud y la educación de los hijos.
2015; Marchena, 2015), con excepción de las "mujeres muestra" (Bareiro et al. 2007). Segundo, las normas culturales indígenas continúan privilegiando a los varones, no obstante se argumente que las relaciones hombre-mujer en la cosmovisión indígena son de complementariedad y armonía (Fundación Rigoberta Menchú, 2003). Reducir la exclusión de género sucede al aceptar y facilitar la reflexión abierta de las mujeres sobre el manejo de los bosques sin anteponer el bien colectivo indígena a las diferencias de género (Ulloa, 2007; Parrado e Isidro, 2014) en el hogar y las estructuras comunitarias.

\section{Resultados y discusión}

1 Pocas mujeres participan en las decisiones sobre el manejo de los bosques comunitarios debido en parte a la débil gobernanza.

La estructura de la gobernanza indígena se divide entre la comunidad y el territorio. La comunidad es la forma de organización tradicional de los pueblos indígenas de la RACCN, y por ley se refiere a su espacio territorial, identidad y formas de organización propia, incluyendo su autoridad comunal. Los Gobiernos Territoriales Indígenas $(\mathrm{GTI})^{3}$ son entes relativamente nuevos creados en el marco de la titulación de tierra comunitaria

3 Un GTI "tiene a su cargo la administración política del territorio, sistemas de educación, salud, economía y deporte. Se elige democráticamente en una asamblea territorial. Sus órganos centrales son: La Junta directiva, conformada por el presidente, vicepresidente, secretario, tesorero, fiscal y un vocal. Sus miembros son electos por un período no mayor a cuatro años, con la opción de ser reelectos una sola vez, ello, sí la asamblea territorial y comunal lo decide" (Hellmann y Unger, 2012 pág 15). 
que tiene lugar en el país desde el año 2003 con la aprobación de la Ley 445 de la propiedad comunal de los pueblos indígenas (Hellmann y Unger, 2012). En el gobierno comunitario y territorial indígena, las autoridades (síndico, wihta y presidente) toman decisiones, gestionan y coordinan con instancias de gobierno, empresas y organizaciones no gubernamentales; también reciben del Estado un porcentaje de impuestos del aprovechamiento de los recursos forestales. En ambas instancias, la representación de mujeres y varones es desigual. La revisión de cargos en tres Gobiernos Territoriales Indígenas (GTI) indica 22 cargos, de los cuales solo 4 son ocupados por mujeres. De 43 integrantes en los cinco gobiernos comunales, 35 son hombres y 8 son mujeres (Castillo, 2015). Los cargos principales de gobierno comunal son ocupados por el síndico y el wihta casi siempre ocupado por varones. A las mujeres se les propone el cargo de tesorera por la capacidad de "cuidar" y "respetar el dinero", pero en la práctica no significa que tienen la libertad para ejercer tales capacidades pues los presidentes del GTI controlan personalmente el dinero que reciben del Estado. La preferencia por una mujer en este cargo es una oportunidad, sin embargo termina siendo un mecanismo para afianzar su rol de "mujer que cuida", mientras que los cargos de síndico, wihta o presidente se identifican con el ejercicio de la toma de decisiones y por ello son los más buscados y ejercidos por los varones.

Durante las actividades de monitoreo participativo, los comunitarios identificaron varias deficiencias y problemas de gobernanza. Por ejemplo, en una comunidad, la mayoría de comunitarios, y en particular las mujeres, aducen no participar y no recibir información sobre las gestiones de sus líderes. Cedeño (2015), en una de las sesiones de monitoreo sobre la participación de las mujeres en la gestión del bosque, escribió: "solo se reúnen el Wihta y el Síndico, ellos contratan a su gente que tienen ya escogida y trabajan sin que la comunidad se dé cuenta de lo que hacen y para que no interfieran en los ingresos económicos que se obtienen por otorgar permisos forestales" (Discusión, comunidad "K", 8 de mayo 2015).

De las personas sin cargos, más mujeres que varones mencionan que se les ve como un obstáculo. "... no nos involucran ... porque si a nosotras nos involucran en las juntas directivas, vamos a evaluarlos y monitorearlos a ellos" (discusión con mujeres miskitu, 22 de mayo de 2015). También se planteó que, "a las mujeres no les da estar el derecho de participar en reuniones donde se permite extraer madera ... porque ellas no callarían lo que pasaría, informarían a toda la familia comunal; no esconderían las malas decisiones que toman los líderes para su propio interés. Cuando sienten que las mujeres están por escuchar o intervenir en temas relacionado con eso, las autoridades se reúnen de noche con un pequeño grupo de personas de su junta y cuando se sienten amenazados expresan: ¿cómo es posible que las mujeres nos manden?, ellas no tienen ni voz, ni voto sobre las decisiones que nosotros tomemos..." (Discusión grupal en actividad de monitoreo, 9 Mayo 2015). Opiniones y reflexiones como las anteriores emergieron del proceso de monitoreo que abrió espacios para discutir sobre las preocupaciones de la gobernanza interna y el rol de las mujeres en el manejo colectivo de los recursos naturales, un proceso tensionado por las actitudes y opiniones de muchos varones que ven en las mujeres falta de información y capacidad para gobernar y manejar sus bosques, pese a que el conocimiento ancestral de las abuelas permitiría readaptar prácticas ancestrales para enfrentar la destrucción de los bosques (Cunningham, 2011, Evans, et al., en proceso de publicación).

Adicionalmente, el conflicto por la tierra indígena provocado por la invasión de "colonos"4 en los años recientes (2014 y 2015) es un factor que se percibe desfavorable para las mujeres y contribuye a cerrar espacios logrados para ellas. Como señalan Marchena (2015), Pikitle (2015) y Davis et al. (2016), aunque el conflicto afecta a todos, se argumenta que las mujeres "no pueden estar ahí", dado que "compete a los varones proteger a la familia y a la comunidad", así de forma "persuasiva ellas ceden su lugar a los hombres".

\section{Normas culturales que privilegian lo colectivo pero desfavorecen a las mujeres.}

En el proceso de monitoreo, así como en otros momentos de la investigación participativa, muchos varones argumentaron que no hay exclusión de la mujer, sino que son ellas quienes no aceptan participar o asumir un cargo, poniendo el problema en las mujeres. La reflexión sobre la incorporación de las mujeres afloró ideas contradictorias: por un lado, para que una mujer ocupe un cargo en las estructuras comunitarias, esa mujer debería ser soltera para que no se vea en el dilema de no cumplir por las responsabilidades ante los hijos, el marido y el hogar. Por otro lado, en algunas comunidades se argumenta que la mujer, para ser líder, debería casada y contar con el permiso del marido (McLean, 2016, comunicación personal).

Paradójicamente, las solteras a quienes se les asigna el criterio de selección, suelen ser mujeres jóvenes, razón por la cual se les señala de no tener el conocimiento ni habilidad necesaria para ejercer liderazgo en una cultura que valora el conocimiento a partir de los años y la experiencia vivida. Entre más años tiene una persona, se considera con mayor conocimiento y por tanto con derecho a opinar y ser escuchada; tal es el caso de los ancianos y ancianas. No obstante, ser anciana supone también enfrentar restricciones de movilidad fuera del hogar, la comunidad y el territorio debido a la edad. Así, ni ser joven ni ser anciana son criterios que las hacen merecedoras de ocupar cargos y opinar sobre las decisiones respecto al aprovechamiento forestal. A las mujeres casadas se les restringen sus derechos cuando se les hace saber que ellas pueden ocupar cargos solo si cuentan con el permiso del marido y del resto de líderes masculinos. Esta forma de pensar constituye una barrera creada culturalmente para acentuar las diferencias entre hombres y mujeres e inhibir a las mujeres de su libre participación, lo que a la vez funciona como mecanismo de resistencia al cambio que demandan las mujeres para ser tomadas en cuenta.

En este escenario, las normativas culturales respecto a los roles asignados a ellas y ellos, también se entrelazan con otros factores que actúan en contra de las mujeres. Por ejemplo, la insistencia de líderes varones de participar en los espacios de

4 "Colono" se les llama a personas no originarias de las comunidades o pueblos indígenas de las Regiones Autónomas de la Costa Caribe de Nicaragua que toman posesión de tierras en territorio indígena sin o con el consentimiento de la comunidad o sus líderes. 
discusión que se facilitaron en el marco del monitoreo, mostró el afán de algunos líderes de controlar las opiniones de las mujeres a quienes se les señalaba de no saber de lo que hablaban o se les indicaba que era mejor callar. Observamos también el caso de una mujer indígena que fue electa para ser jueza comunitaria y enfrentó el cuestionamiento hasta de sus mismos parientes por su supuesta incapacidad de impartir justicia; incluso pasó unos meses en la cárcel bajo la acusación de cometer actos ilícitos. En contraposición, ningún juez comunitario ha enfrentado un proceso de descrédito y cárcel, aunque haya cometido los mismos actos ilícitos de los que se le acusó a ella. En las recientes elecciones de algunas autoridades comunitarias y territoriales se imponen opiniones por parte de los varones de excluir a las mujeres bajo el argumento de que ellas no pueden resolver los problemas que enfrenta la comunidad para defender su territorio por prácticas ilícitas sobre la tierra y la presencia de no-indígenas. No obstante, la esperanza de cambio se manifiesta cuando en una comunidad algunas mujeres han sido electas recientemente en los principales cargos, es decir como vicesíndica y como wihta. En opinión de uno de los reverendos de la iglesia en la comunidad, ellas están dando muestras de que se coordinan mejor que los varones en el trabajo para el bien de la comunidad.

\section{Conclusiones}

La escasa representación de las mujeres en la toma de decisiones sobre el manejo de los bosques comunitarios en las estructuras comunitarias y territoriales yace en un pensamiento que relega a la mujer al espacio doméstico, al margen de la gobernanza comunitaria que es ejercida desde una visión masculina, poco cuestionada. En este escenario, cualquier esfuerzo orientado a mejorar las condiciones de vida de las familias indígenas, debe considerar esta situación con el fin de evitar que se agraven los procesos de inequidad y desigualdad de género. Si bien es necesario pensar en términos de la urgente necesidad para la inclusión de más mujeres en la gobernanza comunitaria, es preciso tener en consideración que la inclusión en sí misma no garantiza un cambio en las relaciones de hombres y mujeres en la comunidad indígena. Se necesitan espacios para la reflexión, sensibilización y concientización de las mujeres - y de los hombres - sobre el rol de ellas en el manejo de los bosques, espacios que se pueden abrir a partir de monitorear su participación.

Revertir el problema de la exclusión y promover un proceso de inclusión de mujeres mayangnas y miskitu en el espacio rural precisa reconocer que existen aspectos culturales y de poder subyacentes que, aunque incrustados en el pensamiento y en la práctica cotidiana, están amparados por normativas que generan inequidad. Las mujeres indígenas rurales necesitan no solo ser incluidas en mayor número dentro de las estructuras comunitarias en cargos importantes del liderazgo; también se necesitan espacios de mujeres para reflexionar sobre sus preocupaciones en el contexto de presión y conflictos por la tierra y los bosques, el riesgo de la inseguridad alimentaria, la deforestación, la contaminación de las fuentes de agua y los problemas internos de gobernanza. El desafío mayor es fortalecer la organización comunitaria trascendiendo las demandas legítimas del pueblo indígena como colectividad, para que las preocupaciones de las mujeres respecto al deterioro de los bosques y el manejo de los recursos de la comunidad no queden supeditadas o postergadas, sino que sean parte de las soluciones que se necesitan gestar en el hogar y en otros espacios de vida comunitaria. El monitoreo participativo ha sido un espacio en el que se abrieron esperanzas de cambio no solo para las mujeres, sino también para los varones que no ocupan cargos. De esta manera, incrementar y fortalecer la participación de las mujeres es un insumo importante para fortalecer la gobernanza comunitaria.

\section{Agradecimientos}

Agradecemos en primera instancia a las mujeres miskitu y mayangnas de las comunidades que participaron en el proceso de monitoreo. También agradecemos la colaboración inicial de Sandra Davis, así como los comentarios y observaciones realizados por Fernanda Soto, Jennifer Casolo, David Kaimowitz y Melba McLean. Esta investigación fue financiada por la Agencia de Desarrollo Austríaca (ADA). Las opiniones expresadas en el presente documento pertenecen a los autores y no reflejan necesariamente los puntos de vista de CIFOR ni de las instituciones a las cuales se encuentran vinculados.

\section{Referencias}

Bareiro L, Soto C, y Soto L. (2007). La inclusión de las mujeres en los procesos de reforma politica en América Latina. Washington: Unidad para la Igualdad de Género en el Desarrollo, Banco Interamericano de Desarrollo.

Castillo M. (10 de noviembre de 2015). Entrevista sobre el registro de miembros de los gobiernos territoriales indígenas que tiene Cejudhcan. (S. Davis, entrevistador)

Cedeño K. (2015). Informe de la sesión de monitoreo sobre los instrumentos de la gobernanza comunitaria. Sesión para la evaluación de los instrumentos de monitoreo. Comunidad Kisalaya: Nitlapan.

Cunningham R. (2011). Rol de Mujeres Indígenas.

Conocimientos tradicionales y prácticas. Herramientas. En: D. Mairena, Ed. Conocimiento Tradicional, Mujeres Indígenas Y Bosques: Estudios de Caso En La Costa Caribe de Nicaragua. Bilwi, CADPI/Wangki Tangni.

Davis S, Barbeyto A, López S, Marchena R y Pikitle A. (2016). Construyendo cambios desde las mujeres: Género, Cosmovisión y Bosque en comunidades Miskitu y Mayangna. Managua: Nitlapan-CIFOR.

Evans K, Flores S, Larson A, Muller P, Marchena R, y Pikitle A. (en proceso de publicación). Challenges for women's participation in communal forest, experiences from Nicaragua's indigeneous territories.

Fundación Rigoberta Menchú. (2003). Memoria Primera Cumbre de Mujeres Indígenas de América. México.

Hellman, T, y Unger J. (2012). Institucionalización de los Gobiernos Territoriales Indigenas (GTI). La experiencia del AVAR Indígena del proyecto INAP en la RAAN, Nicaragua. Obtenido de www.masrenace: http://masrenace.wikispaces.com/file/ view/Sistematizacion+AVAR_borrado+final+2012.pdf 
Mairena E, Lorio G, Hernández X, Wilson C, Müller P y Larson AM. 2012 Gender and forests in Nicaragua's indigenous territories: From national policy to local practice. Working Paper 95. CIFOR, Bogor, Indonesia

Marchena R. (2015). Mujeres y hombres Miskitu: La complejidad en la gobernanza comunitaria. El caso de Iltara. Managua: Nitlapan, documento no publicado basado en notas de campo.

Parrado S, e Isidro L. (2014). La paradoja hipócrita.

Problematización de la participación política de la mujer misak. Colombia Internacional 80, enero a abril, 135-170.
Pikitle A. (2015). Mujeres y hombres Mayangnas: La complejidad en la gobernanza comunitaria, el caso de Awastingni. Managua: Nitlapan, documento no publicado basado en notas de campo.

Ulloa A. (2007). Mujeres indígenas, dilemas de género y etnicidad en los escenarios latinoamericanos. En: Donato LM., Escobar E M, Esobar P, Pazmiño A y Ulloa A (eds), Mujeres Indígenas, Territorialidad y Biodiversidad en el Contexto Latinoamericano (pp. 17-33). Bogotá, Colombia: UNAL, Fundación Natura, UICN y UNODC.

\begin{tabular}{|l|ll|}
\hline PROGRAMA DE & $\begin{array}{l}\text { Esta investigación fue realizada por CIFOR como parte del Programa de Investigación de CGIAR sobre } \\
\text { Bosques, Árboles y Agroforestería (CRP-FTA). El objetivo del programa es mejorar el manejo y uso de los }\end{array}$ \\
INVESTIGACIÓN SOBRE & $\begin{array}{l}\text { Bosques, Árboles y } \\
\text { bosques, la agroforestería y los recursos genéticos de los árboles a lo largo del paisaje, desde bosques } \\
\text { hasta plantaciones. CIFOR dirige el programa CRP-FTA en asociación con Bioversity International, CATIE, } \\
\text { CIgroforestería }\end{array}$ & $\begin{array}{l}\text { CIRAD, el Centro Internacional de Agricultura Tropical y el Centro Mundial de Agroforestería. } \\
\text { CGIAR }\end{array}$
\end{tabular}
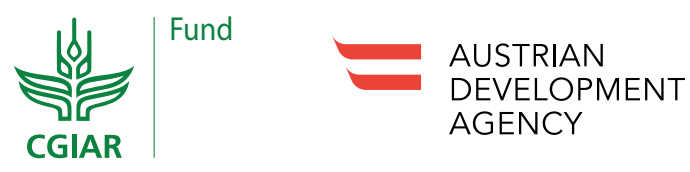
ayudar en el diseño de políticas y prácticas que afectan a los bosques de los países en vías de desarrollo. CIFOR es un miembro del Consorcio CGIAR. Nuestra sede central se encuentra en Bogor, Indonesia, y contamos con oficinas en Asia, África y América Latina. 